\title{
Quality evaluation of strawberry squash stored at ambient temperature
}

\author{
Muhammad Kefayatullah ${ }^{1}$, Haq Nawaz ${ }^{2 *}$, Said Wahab ${ }^{1}$, Mohammad \\ Ayub $^{1}$, Muhammad Zuhair ${ }^{2}$, Muhammad Mehran Anjum², Nawab \\ $\mathrm{Ali}^{2}$, Ashfaq Ayub ${ }^{3}$, Fawad Ahmad ${ }^{1}$ and Daud Ahmad ${ }^{1}$ \\ 1. Department of Food Science and Technology, the University of Agriculture Peshawar-Pakistan \\ 2. Department of Agronomy, the University of Agriculture Peshawar-Pakistan \\ 3. Department of Plant Breeding and Genetics, the University of Agriculture Peshawar-Pakistan \\ *Corresponding author's email: haqnawaz63@aup.edu.pk \\ Citation \\ Muhammad Kefayatullah, Haq Nawaz, Said Wahab, Mohammad Ayub, Muhammad Zuhair, Muhammad \\ Mehran Anjum, Nawab Ali, Ashfaq Ayub, Fawad Ahmad and Daud Ahmad. Quality evaluation of strawberry \\ squash stored at ambient temperature. Pure and Applied Biology. Vol. 8, Issue 1, pp397-403. \\ http://dx.doi.org/10.19045/bspab.2018.700199
}

\begin{tabular}{llll}
\hline \hline Received: 12/10/2018 & Revised: 03/12/2018 & Accepted: 06/12/2018 & Online First: 08/12/2018 \\
\hline \hline
\end{tabular}

\section{Abstract}

The research work was conducted to find out the effect of sodium benzoate and potassium sorbate on the overall quality of strawberry squash, stored at ambient temperature for 90 days. The research samples were numbered as $\mathrm{T}_{1}$ (strawberry squash without preservative), $\mathrm{T}_{2}(0.1 \%$ sodium benzoate $), \mathrm{T}_{3}(0.1 \%$ potassium sorbate $), \mathrm{T}_{4}(0.05 \%$ sodium benzoate + $0.05 \%$ potassium sorbate), $\mathrm{T}_{5}(0.075 \%$ sodium benzoate $+0.025 \%$ potassium sorbate $)$. The samples were studied for $\mathrm{pH}$, TSS, \% acidity, ascorbic acid, reducing sugar, non-reducing sugar and organoleptic evaluation (color, flavor, taste and overall acceptability). A decrease was observed in $\mathrm{pH}$ (2.86 to 1.92$)$, TSS (49.50 to 42.76), ascorbic acid content (41.75 to $24.74)$, non-reducing sugar (26.12 to 24.47 ) and an increase in \% acidity (1.31 to 1.97 ) and reducing sugar (10.40 to 11.85$)$. During sensory evaluation, the sample $\mathrm{T}_{4}(0.05 \%$ sodium benzoate $+0.05 \%$ potassium sorbate) was found most acceptable. Statistical analysis showed that the treatments and storage intervals had a significant $(\mathrm{P}<0.05)$ effect on the physicochemical and organoleptic analysis of strawberry squash.

Keywords: Ambient temperature; Benzoate; Evaluation; Physicochemical Analysis;

Strawberry squash; Sorbate

\section{Introduction}

Strawberry (Fragaria spp.) belongs to kingdom Plantae and is an herbaceous perennial member of family "Rosaceae", the most widely grown berry of the world. There are more than 600 varieties of strawberries which differ in flavor, texture and size. Strawberry can be identified by its red flesh that has yellow seeds piercing its surface and a small, regal, green leafy cap and stem which adorn its crown [1]. In Latin it is referred to as "fragra" while in French, Italian and Spanish it is referred to as "Fraise" or "Fragrant berry". About 250 years ago strawberry came from two different countries, North and South America and then brought to Europe. It is grown in most part of the world but is extensively grown in USA, Japan, Mexico, Italy and Labenon [2].

Due to its delicious flavor and attractive color the consumer's demand for the fruit is increasing not only in Pakistan but also in other parts of the world. Strawberry fruit is mainly consumed as fresh, but is also used in processed form as cooked and 
sweetened preserves i.e. jams, jellies and frozen whole berries. The sweetened juice extract or flavoring is used in making variety of other processed products [3]. Potassium sorbate is also worn in several special concern produce to retard the growth of micro-organisms for ledge. Sodium benzoate is a bacteriostatic and fungistatic preservative underneath acidic environment and is mostly worn in acidic foods like salad dressings (vinegar), carbonated munchies (carbonic acid) and condiments. It can as well be originate in alcohol based mouth wash and in cough syrups. Labeling of this preservative reads as "sodium benzoate" on an ingredient statement or E-211 [4].

Strawberry also contains phyto-nutrients and antioxidants which fight free radicals. The non-reducing sugars on the storage decreases, undergoes inversion changed into reducing sugars. Strawberries also provide an excellent source of vitamin $\mathrm{K}$, riboflavin, vitamin $\mathrm{B}_{5}$, vitamin $\mathrm{B}_{6}$, magnesium, potassium, copper, manganese as well as folic acid and omega 3 fatty acids [5].

$\mathrm{pH}$ of strawberry ranges from 3.27-3.86 which stabilizes color. Titratable acidity ranges from 0.51-1.35 g/100g. Malic and Citric acids are primary organic acids which contribute to flavor. Total soluble solids ranges between $8.0-11.5 \%$ and are ideal for juice concentrate required in the market. A soluble solid per acid ratio 8.52$13.7 \mathrm{~g}$ is a balance of sweetened flavor. Strawberry per $100 \mathrm{~g}$ contains $90.9 \mathrm{~g}$ water, $0.7 \mathrm{~g}$ protein, $7.7 \mathrm{~g}$ carbohydrates, $0.3 \mathrm{~g}$ fats, $16.0 \mathrm{mg}$ calcium, $0.4 \mathrm{mg}$ iron, $13.0 \mathrm{mg}$ magnesium, $1.0 \mathrm{mg}$ sodium, $58.8 \mathrm{mg}$ vitamin $\mathrm{C}$ and $12.0 \mathrm{IU}$ vitamin $\mathrm{A}$ [6].

Keeping in view the perishability of strawberry fruit, the present study was designed to prepare a value added product from strawberry i.e. squash which will be available throughout the year in a market. The farmers will be benefitted while getting proper return for their produce.

\section{Materials and methods}

Sound and healthy strawberries of proper size and optimum maturity were purchased from the local market of Peshawar and brought to the laboratories of Food Technology Center, Pakistan Council of Scientific and Industrial Research (PCSIR) Peshawar, where a research work was conducted. After sorting and washing, the stalks of strawberry fruits were removed by using stainless steel knives. The pulp was extracted with the help of a pulper and strained through muslin cloth and used for squash preparation in the ratio of $1: 1: 1$. Citric acid was also added to adjust the acidity to $1 \%$ along with $0.05 \%$ Carboxy Methyl Cellulose (CMC) to avoid separation. The treatments were made as $\mathrm{T}_{1}=$ Strawberry squash without preservative (Control), $\mathrm{T}_{2}=$ Sodium benzoate $0.1 \%, \mathrm{~T}_{3}=$ Potassium sorbate $0.1 \%, \mathrm{~T}_{4}=$ Sodium benzoate $0.05 \%+$ Potassium sorbate $0.05 \%$ and $\mathrm{T}_{5}=$ Sodium benzoate $0.075 \%+$ Potassium sorbate $0.025 \%$.

\section{Packaging and storage of squash}

The ready squash samples were packed in sterilized $250 \mathrm{ml}$ capacity clear glass bottles and were kept at ambient temperature for physico-chemical and sensory analysis at intervals of 15 days for a total period of 3 months.

\section{Chemical analysis}

Ascorbic acid was determined by the direct colorimetric method using 2,6dichlorophenol-indophenols as decolorizing agent by ascorbic acid in sample extract and in standard ascorbic acid solution [7]. Acidity was determined by standard method of [7]. Inolab digital $\mathrm{pH}$ meter was used for $\mathrm{pH}$ determination. Reducing and non-reducing sugars were determined by Lane Eynon method [7]. The total soluble solids TSS were determined by using Abbe refractometer at room temperature [7].

\section{Sensory evaluation}

A panel of judges selected from students of food science department evaluated the product fortnightly for color, flavor, taste and overall acceptability by the method of 
[8] using a scale from 1 to 9 , where 1 represents extremely disliked and 9 represents extremely liked.

\section{Statistical analysis}

The data obtained was subjected to statistical analysis using RCBD (Randomized Complete Block Design) and the means were compared by using LSD (Least Significant Difference) test [9]. For all the analysis, the alpha error was set at $0.05 \%$.

\section{Results and discussion Chemical analysis}

The statistical analysis indicated that storage intervals and temperature had a significant effect on $\mathrm{pH}$ of all the samples .The mean $\mathrm{pH}$ values of all the samples decreased from 2.86 to 1.92 during storage. Maximum percent decrease was recorded in $\mathrm{T}_{1}(47.90 \%)$, while minimum in $\mathrm{T}_{4}(24.83 \%)$ (Table 1). These results are in close conformity with the findings of [10] who observed a decrease in $\mathrm{pH}$ of citrus fruit beverage.

The data showed that different treatments and storage intervals had a significant effect on ascorbic acid content of different samples. There was a gradual decrease in ascorbic acid of strawberry squash from $41.75 \mathrm{mg}$ to $24.74 \mathrm{mg}$. Minimum percent decrease was recorded in sample $\mathrm{T}_{4}$ $(32.53 \%)$ and maximum in $\mathrm{T}_{1}(58.63 \%)$ (Table 2). In a similar study [11] reported a decrease in ascorbic acid in juices during storage. These results are also in agreement with the findings of [12] who recorded a change in Chalder variety of strawberries stored at $4{ }^{0} \mathrm{C}$.

Acidity of samples ( $\mathrm{T}_{1}$ to $\left.\mathrm{T}_{5}\right)$ were gradually increased during three months of storage. The mean values increased from 1.31 to $1.97 \%$. Maximum mean values were recorded in sample $\mathrm{T}_{1}$ (1.81) followed by $\mathrm{T}_{5}$ (1.62), while minimum mean values were observed in sample $\mathrm{T}_{4}$ (1.53) followed by $T_{2}$ (1.59). During storage maximum increase was observed in $\mathrm{T}_{1}(74.81 \%)$, while minimum increase was observed in $\mathrm{T}_{4}(39.69 \%)$ (Table 3). These results are in agreement with the findings of [13] who reported an increase in acidity of strawberry juice during storage. These findings are in close confirmity with the results of [14] who observed an increase in acidity of kinnow juice storage.

The analysis of the data showed that different treatments and storage intervals had a significant effect on Total Soluble Solids of strawberry squash. Maximum mean values were recorded in $\mathrm{T}_{4}$ (46.69) followed by $\mathrm{T}_{3}$ (46.56), while minimum mean values were recorded in $\mathrm{T}_{1}$ (44.71) followed by $\mathrm{T}_{5}$ (45.87). The results indicated a gradual decrease in TSS of all the samples. Maximum decrease was observed in $\mathrm{T}_{1}(17.78 \%)$ and minimum in $\mathrm{T}_{4}(11.71 \%)$ (Table 4). These findings are in close conformity with the research work of [15] who showed a significant decrease in TSS of strawberry syrup during storage. Results showed that reducing sugars increased in all the samples from 10.40 to $11.85 \%$ during three months of storage. The treatments and storage intervals had a significant effect on reducing sugar of strawberry squash. Maximum percent increase was observed in $\mathrm{T}_{1}(18.56 \%)$ while minimum percent increase was noted in $\mathrm{T}_{2}(10.23 \%)$ (Table 5). These results are in agreement with [16] who showed an increase in glucose and fructose contents in strawberry fruits. Similar findings were also reported by [17] that non reducing sugars of drinks is converted into reducing sugars during storage.

The non-reducing sugars decreased in all the samples from 26.12 to $24.47 \%$. The maximum mean value was noted in $\mathrm{T}_{4}$ (25.63), while minimum mean value was observed in $\mathrm{T}_{1}$ (24.74). Minimum percent decrease was noted in $\mathrm{T}_{4}(5.09 \%)$ and maximum in $\mathrm{T}_{1}(9.80 \%)$ (Table 6). These results are confirmed by [16] who suggested that the sucrose content of the fruit convert to glucose and fructose during storage, results in the change of sucrose contents of the juices. Similar trends of decreasing non-reducing sugars 
were also observed by [18] during studies

on mango squash.

Table 1. The pH of strawberry squash during storage at ambient temperature

\begin{tabular}{|c|c|c|c|c|c|c|c|c|c|}
\hline \multirow{2}{*}{ Treatments } & \multicolumn{7}{|c|}{ Storage intervals (Days) } & $\begin{array}{c}\text { \% } \\
\text { Decrease }\end{array}$ & Means \\
\cline { 2 - 10 } & Initial & $\mathbf{1 5}$ & $\mathbf{3 0}$ & $\mathbf{4 5}$ & $\mathbf{6 0}$ & $\mathbf{7 5}$ & $\mathbf{9 0}$ & $\mathbf{2}$ \\
\hline $\mathbf{T}_{\mathbf{1}}$ & 2.86 & 2.56 & 2.17 & 2.02 & 1.88 & 1.68 & 1.49 & 47.90 & $\begin{array}{c}2.09 \mathrm{c} \\
\pm 0.48\end{array}$ \\
\hline $\mathbf{T}_{\mathbf{2}}$ & 2.86 & 2.68 & 2.51 & 2.32 & 2.20 & 2.02 & 1.97 & 31.12 & $\begin{array}{c}2.36 \mathrm{~b} \\
\pm 0.34\end{array}$ \\
\hline $\mathbf{T}_{\mathbf{3}}$ & 2.86 & 2.70 & 2.55 & 2.39 & 2.23 & 2.09 & 1.93 & 32.52 & $\begin{array}{c}2.39 \mathrm{~b} \\
\pm 0.33\end{array}$ \\
\hline $\mathbf{T}_{\mathbf{4}}$ & 2.86 & 2.71 & 2.63 & 2.51 & 2.42 & 2.29 & 2.15 & 24.83 & $\begin{array}{c}2.51 \mathrm{a} \\
\pm 0.24\end{array}$ \\
\hline $\mathbf{T}_{\mathbf{5}}$ & 2.86 & 2.69 & 2.57 & 2.44 & 2.31 & 2.19 & 2.07 & 27.62 & $\begin{array}{c}2.45 \mathrm{ab} \\
\pm 0.28\end{array}$ \\
\hline Means & $\begin{array}{c}2.86 \mathrm{a} \\
\pm 0.00\end{array}$ & $\begin{array}{c}2.66 \mathrm{~b} \\
\pm 0.64\end{array}$ & $\begin{array}{c}2.48 \mathrm{c} \\
\pm 0.18\end{array}$ & $\begin{array}{c}2.33 \mathrm{~d} \\
\pm 0.19\end{array}$ & $\begin{array}{c}2.20 \mathrm{e} \\
\pm 0.20\end{array}$ & $\begin{array}{c}2.05 \mathrm{f} \\
\pm 0.23\end{array}$ & $\begin{array}{c}1.92 \mathrm{~g} \\
\pm 0.25\end{array}$ & & \\
\hline
\end{tabular}

Figures with different small letters are significantly different $(\mathrm{P}<0.05)$ from each other in their respective column

Table 2. Ascorbic acid (mg/100g) of strawberry squash during storage at ambient temperature

\begin{tabular}{|c|c|c|c|c|c|c|c|c|c|}
\hline \multirow{2}{*}{ Treatments } & \multicolumn{7}{|c|}{ Storage intervals (Days) } & $\begin{array}{c}\text { \% } \\
\text { Decrease }\end{array}$ & Means \\
\cline { 2 - 10 } & Initial & $\mathbf{1 5}$ & $\mathbf{3 0}$ & $\mathbf{4 5}$ & $\mathbf{6 0}$ & $\mathbf{7 5}$ & $\mathbf{9 0}$ & $\mathbf{2}$ \\
\hline $\mathbf{T}_{\mathbf{1}}$ & 41.75 & 36.54 & 31.33 & 29.57 & 25.41 & 20.71 & 17.27 & 58.63 & $\begin{array}{c}28.94 \mathrm{c} \\
\pm 8.60\end{array}$ \\
\hline $\mathbf{T}_{\mathbf{2}}$ & 41.75 & 39.70 & 37.81 & 35.35 & 32.21 & 29.25 & 27.33 & 34.54 & $\begin{array}{c}34.77 \mathrm{ab} \\
\pm 5.40\end{array}$ \\
\hline $\mathbf{T}_{\mathbf{3}}$ & 41.75 & 38.16 & 37.81 & 34.98 & 31.81 & 28.18 & 25.55 & 38.80 & $\begin{array}{c}33.03 \mathrm{ab} \\
\pm 5.81\end{array}$ \\
\hline $\mathbf{T}_{\mathbf{4}}$ & 41.75 & 39.90 & 37.30 & 35.12 & 33.67 & 30.21 & 28.17 & 32.53 & $\begin{array}{c}35.16 \mathrm{a} \\
\pm 4.93\end{array}$ \\
\hline $\mathbf{T}_{\mathbf{5}}$ & 41.75 & 38.60 & 36.60 & 33.12 & 30.78 & 27.20 & 25.40 & 39.16 & $\begin{array}{c}33.35 \mathrm{~b} \\
\pm 6.00\end{array}$ \\
\hline Means & $\begin{array}{c}41.75 \mathrm{a} \\
\pm 0.00\end{array}$ & $\begin{array}{c}38.58 \mathrm{~b} \\
\pm 1.35\end{array}$ & $\begin{array}{c}36.17 \mathrm{c} \\
\pm 2.75\end{array}$ & $\begin{array}{c}33.63 \mathrm{~d} \\
\pm 2.43\end{array}$ & $\begin{array}{c}30.78 \mathrm{e} \\
\pm 3.17\end{array}$ & $\begin{array}{c}27.11 \mathrm{f} \\
\pm 3.75\end{array}$ & $\begin{array}{c}24.74 \mathrm{~g} \\
\pm 4.34\end{array}$ & & \\
\hline
\end{tabular}

Figures with different small letters are significantly different $(\mathrm{P}<0.05)$ from each other in their respective column

Table 3. Percent acidity of strawberry squash during storage at ambient temperature

\begin{tabular}{|c|c|c|c|c|c|c|c|c|c|}
\hline \multirow[b]{2}{*}{ Treatments } & \multicolumn{7}{|c|}{ Storage intervals (Days) } & \multirow{2}{*}{$\begin{array}{c}\% \\
\text { Increase }\end{array}$} & \multirow{2}{*}{ Means } \\
\hline & $\underset{l}{\text { Initia }}$ & 15 & 30 & 45 & 60 & 75 & 90 & & \\
\hline $\mathbf{T}_{1}$ & 1.31 & 1.44 & 1.69 & 1.87 & 1.99 & 2.13 & 2.29 & 74.81 & $\begin{array}{r}1.81 \mathrm{a} \\
\pm 0.35\end{array}$ \\
\hline $\mathbf{T}_{2}$ & 1.31 & 1.30 & 1.49 & 1.62 & 1.73 & 1.81 & 1.95 & 48.85 & $\begin{array}{l}1.57 \mathrm{bc} \\
\pm 0.24\end{array}$ \\
\hline $\mathbf{T}_{3}$ & 1.31 & 1.39 & 1.49 & 1.62 & 1.73 & 1.81 & 1.90 & 45.04 & $\begin{array}{r}1.61 b \\
\pm 0.22\end{array}$ \\
\hline $\mathbf{T}_{4}$ & 1.31 & 1.31 & 1.43 & 1.50 & 1.63 & 1.75 & 1.83 & 39.69 & $\begin{array}{r}1.53 \mathrm{c} \\
\pm 0.20\end{array}$ \\
\hline $\mathbf{T}_{5}$ & 1.31 & 1.39 & 1.47 & 1.61 & 1.73 & 1.84 & 1.90 & 45.02 & $\begin{array}{r}1.62 \mathrm{~b} \\
+0.22\end{array}$ \\
\hline Means & $1.31 \mathrm{f}$ & $1.36 \mathrm{f}$ & $1.51 \mathrm{e}$ & $1.64 \mathrm{~d}$ & $1.76 \mathrm{c}$ & $1.86 \mathrm{~b}$ & $1.97 \mathrm{a}$ & & \\
\hline
\end{tabular}




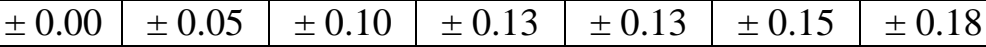

Figures with different small letters are significantly different $(\mathrm{P}<0.05)$ from each other in their respective column

Table 4. TSS of strawberry squash during storage at ambient temperature

\begin{tabular}{|c|c|c|c|c|c|c|c|c|c|}
\hline \multirow[b]{2}{*}{ Treatments } & \multicolumn{7}{|c|}{ Storage intervals (Days) } & \multirow{2}{*}{$\begin{array}{c}\% \\
\text { Decrease }\end{array}$} & \multirow[b]{2}{*}{ Means } \\
\hline & Initial & 15 & 30 & 45 & 60 & 75 & 90 & & \\
\hline $\mathbf{T}_{1}$ & 49.5 & 47.0 & 46.1 & 44.7 & 43.1 & 41.9 & 40.7 & 17.78 & $\begin{array}{l}44.71 \mathrm{c} \\
\pm 3.07\end{array}$ \\
\hline $\mathbf{T}_{2}$ & 49.5 & 47.6 & 46.7 & 45.8 & 44.9 & 43.7 & 43.3 & 12.53 & $\begin{array}{l}45.93 b \\
\pm 2.20\end{array}$ \\
\hline $\mathbf{T}_{\mathbf{3}}$ & 49.5 & 48.5 & 47.7 & 46.8 & 45.3 & 44.7 & 43.4 & 12.32 & $\begin{array}{l}46.56 \mathrm{a} \\
\pm 2.19\end{array}$ \\
\hline $\mathbf{T}_{4}$ & 49.5 & 48.7 & 47.9 & 46.8 & 45.3 & 44.9 & 43.7 & 11.71 & $\begin{array}{l}46.69 \mathrm{a} \\
\pm 2.14\end{array}$ \\
\hline $\mathbf{T}_{5}$ & 49.5 & 47.8 & 46.7 & 45.4 & 44.7 & 43.3 & 42.7 & 13.74 & $\begin{array}{l}45.87 \mathrm{~b} \\
\pm 2.60\end{array}$ \\
\hline Means & $\begin{array}{l}49.50 \mathrm{a} \\
\pm 0.00\end{array}$ & $\begin{array}{l}48.12 b \\
\pm 0.78\end{array}$ & $\begin{array}{l}47.02 \mathrm{c} \\
\pm 0.76\end{array}$ & $\begin{array}{l}45.90 \mathrm{~d} \\
\pm 0.91\end{array}$ & $\begin{array}{l}44.66 \mathrm{e} \\
\pm 0.91\end{array}$ & $\begin{array}{l}43.70 \mathrm{f} \\
\pm 1.21\end{array}$ & $\begin{array}{l}42.76 \mathrm{~g} \\
\pm 1.20\end{array}$ & & \\
\hline
\end{tabular}

Figures with different small letters are significantly different $(\mathrm{P}<0.05)$ from each other in their respective column

Table 5. Reducing sugar percentage of strawberry squash during storage at ambient temperature

\begin{tabular}{|c|c|c|c|c|c|c|c|c|c|}
\hline \multirow{2}{*}{ Treatments } & \multicolumn{7}{|c|}{ Storage intervals (Days) } & $\begin{array}{c}\text { \% } \\
\text { Increase }\end{array}$ & Means \\
\cline { 2 - 10 } & Initial & $\mathbf{1 5}$ & $\mathbf{3 0}$ & $\mathbf{4 5}$ & $\mathbf{6 0}$ & $\mathbf{7 5}$ & $\mathbf{9 0}$ & $\begin{array}{c}11.48 \mathrm{a} \\
\pm 0.66\end{array}$ \\
\hline $\mathbf{T}_{\mathbf{1}}$ & 10.40 & 10.93 & 11.25 & 11.53 & 11.83 & 12.07 & 12.33 & 18.56 & $\begin{array}{c}10.91 \mathrm{~d} \\
\pm 0.44\end{array}$ \\
\hline $\mathbf{T}_{\mathbf{2}}$ & 10.40 & 10.55 & 10.70 & 10.81 & 11.09 & 11.30 & 11.51 & 10.63 & $\begin{array}{c}11.11 \mathrm{c} \\
\pm 0.60\end{array}$ \\
\hline $\mathbf{T}_{\mathbf{3}}$ & 10.40 & 10.67 & 10.89 & 11.09 & 11.37 & 11.57 & 11.79 & 13.36 & $\begin{array}{c}11.06 \mathrm{c} \\
\pm 0.60\end{array}$ \\
\hline $\mathbf{T}_{\mathbf{4}}$ & 10.40 & 10.66 & 10.84 & 11.12 & 11.34 & 11.46 & 11.62 & 11.74 & $\begin{array}{c}11.28 \mathrm{~b} \\
\pm 0.58\end{array}$ \\
\hline $\mathbf{T}_{\mathbf{5}}$ & 10.40 & 10.77 & 11.00 & 11.33 & 11.63 & 11.84 & 11.99 & 15.28 & \\
\hline Means & $\begin{array}{c}10.40 \mathrm{~g} \\
\pm 0.00\end{array}$ & $\begin{array}{c}10.72 \mathrm{f} \\
\pm 0.24\end{array}$ & $\begin{array}{c}10.94 \mathrm{e} \\
\pm 0.25\end{array}$ & $\begin{array}{c}11.18 \mathrm{~d} \\
\pm 0.29\end{array}$ & $\begin{array}{c}11.45 \mathrm{c} \\
\pm 0.29\end{array}$ & $\begin{array}{c}11.65 \mathrm{~b} \\
\pm 0.26\end{array}$ & $\begin{array}{c}11.85 \mathrm{a} \\
\pm 0.25\end{array}$ & & \\
\hline
\end{tabular}

Figures with different small letters are significantly different $(\mathrm{P}<0.05)$ from each other in their respective column

\section{Sensory analysis}

The analysis of the data showed that storage period and treatments had a significant effect on overall acceptability (obtained from color, flavor and taste) of strawberry squash. The mean score of judges decreased from 8.15 to 3.41 .
Maximum mean score of judges was recorded in $\mathrm{T}_{4}(6.20)$ and minimum in $\mathrm{T}_{1}$ (2.60). Maximum decrease was observed in sample $\mathrm{T}_{1}(87.73 \%)$, while minimum increase was observed in $\mathrm{T}_{4}(41.72 \%)$, (Table 7). These results were confirmed by [19]. 
Table 6. Non reducing sugar percentage of strawberry squash during storage at ambient temperature

\begin{tabular}{|c|c|c|c|c|c|c|c|c|c|}
\hline \multirow{2}{*}{ Treatments } & \multicolumn{7}{|c|}{ Storage intervals (Days) } & $\begin{array}{c}\text { \% } \\
\text { Decrease }\end{array}$ & Means \\
\cline { 2 - 10 } & Initial & $\mathbf{1 5}$ & $\mathbf{3 0}$ & $\mathbf{4 5}$ & $\mathbf{6 0}$ & $\mathbf{7 5}$ & $\mathbf{9 0}$ & & \\
\hline $\mathbf{T}_{\mathbf{1}}$ & 26.12 & 25.67 & 25.33 & 24.41 & 24.11 & 23.88 & 23.56 & 09.80 & $\begin{array}{c}24.74 \mathrm{c} \\
\pm 0.97\end{array}$ \\
\hline $\mathbf{T}_{\mathbf{2}}$ & 26.12 & 26.21 & 26.07 & 25.57 & 25.19 & 24.94 & 24.77 & 05.17 & $\begin{array}{c}25.55 \mathrm{ab} \\
\pm 0.60\end{array}$ \\
\hline $\mathbf{T}_{\mathbf{3}}$ & 26.12 & 26.23 & 25.77 & 25.24 & 25.11 & 24.79 & 24.70 & 05.44 & $\begin{array}{c}25.42 \mathrm{ab} \\
\pm 0.62\end{array}$ \\
\hline $\mathbf{T}_{\mathbf{4}}$ & 26.12 & 26.25 & 26.05 & 25.73 & 25.41 & 25.05 & 24.79 & 05.09 & $\begin{array}{c}25.63 \mathrm{a} \\
\pm 0.56\end{array}$ \\
\hline $\mathbf{T}_{\mathbf{5}}$ & 26.12 & 26.01 & 25.73 & 25.29 & 25.01 & 24.69 & 24.53 & 06.09 & $\begin{array}{c}25.34 \mathrm{~b} \\
\pm 0.63\end{array}$ \\
\hline Means & $\begin{array}{c}26.12 \mathrm{a} \\
\pm 0.00\end{array}$ & $\begin{array}{c}26.07 \mathrm{a} \\
\pm 0.24\end{array}$ & $\begin{array}{c}25.79 \mathrm{~b} \\
\pm 0.30\end{array}$ & $\begin{array}{c}25.25 \mathrm{c} \\
\pm 0.51\end{array}$ & $\begin{array}{c}24.97 \mathrm{~d} \\
\pm 0.50\end{array}$ & $\begin{array}{c}24.67 \mathrm{e} \\
\pm 0.46\end{array}$ & $\begin{array}{c}24.47 \mathrm{e} \\
\pm 0.51\end{array}$ & & \\
\hline
\end{tabular}

Figures with different small letters are significantly different $(\mathrm{P}<0.05)$ from each other in their respective column

Table 7. Mean score of judges for overall acceptability of strawberry squash

\begin{tabular}{|c|c|c|c|c|c|c|c|c|c|}
\hline $\begin{array}{c}\text { Treatment } \\
\mathbf{S}\end{array}$ & $\begin{array}{c}\text { Initia } \\
\mathbf{l}\end{array}$ & $\mathbf{1 5}$ & $\mathbf{3 0}$ & $\mathbf{4 5}$ & $\mathbf{6 0}$ & $\mathbf{7 5}$ & $\mathbf{9 0}$ & $\begin{array}{c}\text { \% } \\
\text { Decrease }\end{array}$ & Means \\
\hline $\mathbf{T}_{\mathbf{1}}$ & 8.15 & 4.04 & 2.00 & 1.00 & 1.00 & 1.00 & 1.00 & 87.73 & $\begin{array}{c}2.60 \mathrm{c} \\
\pm 2.69\end{array}$ \\
\hline $\mathbf{T}_{\mathbf{2}}$ & 8.15 & 6.83 & 6.10 & 5.30 & 5.00 & 4.80 & 4.00 & 50.92 & $\begin{array}{c}5.74 \mathrm{ab} \\
\pm 1.40\end{array}$ \\
\hline $\mathbf{T}_{\mathbf{3}}$ & 8.15 & 6.50 & 5.75 & 5.15 & 4.65 & 4.00 & 3.50 & 57.06 & $\begin{array}{c}5.39 \mathrm{~b} \\
\pm 1.58\end{array}$ \\
\hline $\mathbf{T}_{\mathbf{4}}$ & 8.15 & 7.10 & 6.50 & 6.20 & 5.70 & 5.00 & 4.75 & 41.72 & $\begin{array}{c}6.20 \mathrm{a} \\
\pm 1.19\end{array}$ \\
\hline $\mathbf{T}_{\mathbf{5}}$ & 8.15 & 6.66 & 5.65 & 5.10 & 4.70 & 4.10 & 3.80 & 53.37 & $\begin{array}{c}5.45 \mathrm{ab} \\
\pm 1.53\end{array}$ \\
\hline Means & $\begin{array}{c}8.15 \mathrm{a} \\
\pm 0.00\end{array}$ & $\begin{array}{c}6.22 \mathrm{~b} \\
\pm 1.24\end{array}$ & $\begin{array}{c}5.20 \mathrm{c} \\
\pm 1.82\end{array}$ & $\begin{array}{c}4.55 \mathrm{~cd} \\
\pm 2.03\end{array}$ & $\begin{array}{c}4.21 \mathrm{de} \\
\pm 1.84\end{array}$ & $\begin{array}{c}3.78 \mathrm{de} \\
\pm 1.61\end{array}$ & $\begin{array}{c}3.41 \mathrm{e} \\
\pm 1.42\end{array}$ & & \\
\hline
\end{tabular}

Figures with different small letters are significantly different $(\mathrm{P}<0.05)$ from each other in their respective column

\section{Conclusion}

From this study it can be concluded that strawberry squash with $0.05 \%$ sodium benzoate and $0.05 \%$ potassium sorbate was the most acceptable both organoleptically and physicochemically during three months storage. The advantage of this study is to control postharvest losses of strawberry fruits by preparing a shelf stable product.

\section{Authors' contributions}

Conceived and designed the experiments: M Kefayatullah \& S Wahab, Performed the experiments: M Kefayatullah, Analyzed the data: M Kefayatullah \& Haq
Nawaz, Contributed reagents/ materials/ analysis tools: $\mathrm{H}$ Nawaz, $\mathrm{M}$ Ayub, $\mathrm{M}$ Zuhair, MM Anjum, Nawab Ali, F Ahmad, Wrote the paper: D Ahmad \& A Ayub.

\section{References}

1. Esminger AH, Esminger ME, Kondale JE \& Robson JRK (2007). Foods and Nutrition Encyclopedia. Pegus Press. Clovis, California.

2. Childer NF (1983). Strawberry growing. Modern Fruit Science, Gainsesvile. Hort Publ 451-480.

3. Ford A, K Hansen, M. Herrington, J Moisander, S Nottinghum, S Prytz \& 
M Zorin (1997). Subjective and objective determination of strawberry quality. Acta Horticultural 439 (1): 319-323.

4. Kreb \& Mohin (2006). Differences between food group reports of low energy reporters and non low energy reporters on a food frequency questionnaire. $J$ Am Diet Assoc 109(7): 1194-1203

5. USDA (2005). National Agricultural Statistics Service Information.

6. U.S Dept. of Agriculture USDA (2011). Nutrient database for standard references. (WWW. OregonStrawberries. Org).

7. AOAC (2005). Official methods of analysis of the Association of Official Analytical Chemists. $18^{\text {th }}$ ed. Maryland USA.

8. Larmond E (1977). Method for sensory evaluation of food. Canada. Dept of Agriculture Publ 1286: 36-37.

9. Steel RGD, Torrie JH \& Dickey D (1997). Principles and procedures of statistics. A biometrical approach. $3^{\text {rd }}$ ed. Mc Graw Hill Pub. Co., Inc New York, USA.

10. Saleem M (1980). Studies on the preparation of comminuted citrus fruit beverage base. M.Sc. thesis. Agricultural University Faislabad.

11. Sandhu KS \& Singh S (2001). Studies on the factors affecting the physicochemical and organoleptic properties of kinnow juices. J Food Sci Tech 38 (3): 266-269.

12. Nunes MCN, Morais MB, Breecht JK \& Sargent SA (1995). Qualilty of strawberries after storage in controlled atmospheres at above optimum storage temperatures. Proceedings of the Florida State Hort Soc Pub 108: 273-278.

13. Ayub M,Ullah J, Muhammad A \& Zeb A (2010). Evaluation of strawberry juice preserved with chemical preservatives at refrigeration temperature. Int J Nut Metab 2(2): 027-032.

14. Rodriqo D, Arranz JI, Koch S, Frigola A, Rodrigo MC, Esteve MJ, Calvo C \& Rodriqo M (2003). Physicochemical characteristics and quality of refreigarated spinach orange-carrot juices and influence of storage conditions. J Food Sci 68(6): 2111-2116.

15. Khan MM (2010). Preservation of strawberry syrup with benzoate and sorbate. M.Sc. thesis. Deptt. of Food Tech., Univ. Agri., Peshawar.

16. Ruiz-Nieto A, Lopez AJM, Lopez MR, Lopez MJ, Medina JJ, Scheer HAT, Lieten F \& Dijkstra J (1997). Analysis of sucrose's from strawberry cultivars of commercial interest contents evaluation. Proceedings of the third international strawberry symposium, Veldhoven, Netherlands, 29 Vol 2. Acta Hort 439: 663-667.

17. Pruithi JS, Manan JK \& Teotia MS (1984). Studies on the utilization of kinnow and malta oranges. J Food Sci Tech 21(3): 123-127.

18. Gilani, S. A, Y. Fujii, Y, Z. K. Shinwari, M. Adnan, A. Kikuchi \& K. N. Watanabe (2010). Phytotoxic studies of medicinal plant species of Pakistan. Pak J Bot 42(2): 987-996.

19. Kinh Shearer AEH, Dunne CP \& Hoover DG (2001). Preparation and preservation of apple pulp with chemical preservatives and mild heat. J Food Prot 28 (6): 111-114. 